\title{
Channeling Radiation Characteristics of Positrons Channeled in Perfect Single-Walled Carbon Nanotubes
}

\author{
M. K. Abu-Assy, M. Y. El-Ashry and M. H. Ali \\ Physics department, Faculty of Science, Suez-Canal University, Ismailia 41522, Egypt.
}

\begin{abstract}
We investigate the channeling radiation characteristics of positrons channeled in single-walled carbon nanotubes (SWCNTs). The channeled positrons move along the nanotube axis, (z-axis), with energies (10-500) MeV. This study covers SWCNTs with different chiral indices $(n, m)$. The energy eigenvalues, in a plane normal to the nanotube axis (xy-plane), of relativistic positron channeled through SWCNTs has been used to calculate the emitted photon energy in the forward direction, i.e., channeling radiation. Also, the calculations covered both the wave length of the emitted radiation and the maximum number of bound states of the channeled positrons as a function of the nanotube radius at different incident energies. The results of the calculations showed that, in this energy range, the emitted radiation energy lies in the X-ray band and as the energy of the incident positron increase, the emitted radiation energy increase towards the gamma-ray energy band and accordingly, the wave length decrease towards the gamma-ray band for the same transition $\Delta E=E_{n+1}-E_{n}$. For all values of positron energy, the wave length of the emitted radiation was found to be a decreasing function of the nanotube radius while the maximum number of bound states of the channeled positrons is an increasing function of the nanotube radius for the different types of SWCNTs namely, zigzag, armchair and chiral.
\end{abstract}

Keywords: Channeling Radiation; Carbon nanotubes; Relativistic positron beams.

\section{Introduction}

In the case of channeling of relativistic particles through single-walled carbon nanotubes(SWCNTs), the channeled particles move along the nanotube axis, ( $z$-axis) and is influenced by a transverse potential field results from atomic rows $N$ form the nanotubesurface as illustrated in Fig.1, where $N$ is determined according to the nanotube chiral indices $(n, m)$, with $N=(2 / q)\left(n^{2}+n m+m^{2}\right)$, where $q=\operatorname{gcd}(2 m+n, 2 n+m)$ denotes the greatest common divisor of its arguments. Due to this field, the channeled charged particles execute a periodic motion in a definite eigenstates, and radiation results from spontaneous transitions between these states.This radiation was predictedby Kumakhov [1]in the case of axial and planar channeling of charged particles in crystals and was called channeling radiation. He also developed the theory of this phenomena [1,2].

One of the most important applications of carbon nanotubes is the channeling of charged particles through carbon nanotubes (CNTs). The first theoretical study of particle channeling in CNTs appeared in the work of Klimov and Letokhov [3], who showed that relativistic positrons and electrons can emit, respectively monochromatic hard X-rayand gamma-ray when channeled in CNTs. Dedkov [4] gave qualitative arguments showing that CNTs should provide more favorable conditions for ion channeling than single crystals, such as longer dechanneling length due to wider spaces with low electron densities, lower ion energy needed for channeling, as well as larger critical angles for channeling and bending of ion beams, in addition, the channeling potential wells of nanotubes are sufficiently deep and broad to allow an efficient capture of positive particle beam in channeling states.

In this work, we investigate the channeling radiation characteristics of positrons channeled in SWCNTs. The channeled positrons move along the nanotube axis, with energy range (10-500) MeV. This study covers the three known kinds of SWCNTs i.e., zigzag, armchair and chiral with different chiral indices $(n, m)$ to investigate the effect of channeling potential well on the eigenstates of the channeled positrons. The energy eigenvalues, in a plane perpendicular to the nanotube axis ( $x y$-plane), of relativistic positron channeled through SWCNTs has beencalculatedand then used in calculations of the emitted photon energy in the forward direction, i.e., channeling radiation. Also, the calculations covered both the wave length of the emitted radiation and the maximum number of bound states of the channeled positrons as a function of the nanotube radius at different incident energies for the channeled positrons. The results of the calculations showed that, in this energy range of the channeled positrons, the emitted radiation energy lies in the X-ray band and as the energy of the incident positron increase, the emitted radiation energy increase towards the gamma-ray energy band and accordingly, the wavelength decrease towards the gamma-ray band for the same transition. 


\section{Radiation Characteristics from Channeled Positrons}

The channeled positrons are assumed to move along the nanotube axis, (z-axis), as illustrated in Fig.1. The motion of the channeled positron in SWCNT is governed by thenanotube channeling potential i.e., the potential in a plane perpendicular to the tube axis (xy-plane). In previous work [5], the nanotube channeling potential has been calculated according to the continuum model approximation given by Lindhard [6], and by using the atomic interaction potential as given by Moliere [7],for the case of an axial channeling in single crystals. The calculated channeling potential was found to be a one-dimensional function of the distance $\rho$ from the nanotube center in a planenormal to the tube axis and could be fitted reasonably tothe following expression:

$$
U(\rho)=a_{1}+b_{1} e^{c_{1} \rho},
$$

where the fitting parameters $a_{l}, b_{l}$ and $c_{l}$ are given in Table 1 for the carbon nanotubes under consideration.

The energy eigenvalues of the channeled positrons has been calculated by using the WKB method for a particle constrained to move between two classical turning points $\rho_{1}$ and $\rho_{2}$. According to this approximation andby using Eq. (1) for the nanotube channeling potential, the energy eigenvalues of the channeled positron can be calculated from the following condition [8]:

$\int_{\rho_{1}}^{\rho_{2}} p d \rho=\left(n+\frac{1}{2}\right) \pi \hbar, \quad n=0,1,2, \ldots$.

where $p=\left[2 m_{0} \gamma\left(E-\left(a_{1}+b_{1} e^{c_{1} \rho}\right)\right)\right]^{1 / 2}$, is the classical linear momentum, $m_{0}$ is the positron rest mass, $\gamma$ is the relativistic correction and $\hbar=h / 2 \pi$ where $h$ is Planck's constant.

From Eq. (2), we can get the energy eigenvalues and the maximum number of bound states respectively as:

$$
E_{n}=a_{1}+\left(\frac{1}{2 m_{0} \gamma}\right)\left(\frac{c_{1} \pi \hbar}{2 \ln 2}\right)^{2}\left(n+\frac{1}{2}\right)^{2}
$$

and

$$
n_{\max }=\left(\frac{2 \ln 2}{c_{1} \pi \hbar}\right)\left[2 m_{0} \gamma\left(E_{\max }-a_{1}\right)\right]^{1 / 2}-0.5
$$

where $E_{\max }$, is the potential at the turning points, that is

$E_{\text {max }}=a_{1}+b_{1} e^{c_{1} s}, s=R-a$, is the screening length, $R=d / 2$ being the nanotube radius and $a=\left(9 \pi^{2} / 128 z_{2}\right)^{1 / 3} a_{0}$, is the Thomas-Fermi screening radius; $a_{0}=0.529 \AA$ is the Bohr radius, $z_{2}$ is the charge number of the target atoms, and $e$ is the elementary charge

The emitted photon energy in the forward directionis given by:

$$
h v=2 \gamma^{2}(\Delta E) \text {, }
$$

where $\Delta E$, is calculated by using Eq. (3), is the energy difference between the successive initial and final states of the channeled positron and $v$ is the frequency of the emitted radiation $[9,10]$. The wave length of the emitted radiation $\lambda$, is calculated by using Eq. (5) as follows:

$$
\lambda=\frac{c h}{2 \gamma^{2}(\Delta E)},
$$

where, $c \approx 3 \times 10^{10} \mathrm{~cm} / \mathrm{sec}$ is the speed of light.

\section{Computational Results and Discussion}

To illustrate the channeling radiation characteristics, the emitted photon energies due to different allowed transitions were plotted for Moliere potentials for positrons with $\mathrm{E}=50 \mathrm{MeV}$ channeled in in single wall carbon nanotubes (armchair, chiral, zigzag) are presented the emitted photon energy in the forward direction due to the allowed transitions $n,(n-1)$ in Fig. $(1,2$, and 3$)$ for different chiral indices $(n, m)$ of single wall carbon nanotubes respectively.

The total emitted photon energy due to the new transitions $\mathrm{n},(\mathrm{n}-1)$ between higher quantum states are presented in Fig. (4, 5, and 6) for (armchair, chiral, zigzag) respectively as a function of positron energy up to 50 $\mathrm{MeV}$.

The relation between the maximum number of bound states, nmax of the channeled positrons as a function of the nanotube radius at different incident energies (50, 100, and 500) MeV as shown in Fig. (7, 8, and 
9) respectively. The relation between the radius and wavelength in (zigzag, chiral, armchair) single wall carbon nanotubes at (50, 100, and 500) $\mathrm{MeV}$ as shown in Fig. (10, 11, and 12) respectively. This results of the calculations showed that, in this energy range, the emitted radiation energy lies in the X-ray band and as the energy of the incident positron increase, the emitted radiation energy increase towards the $\gamma$ - energy band and accordingly, the wave length decrease towards the $\gamma$-band for the same transition $\Delta E=E_{n+1}-E_{n}$. For all values of positron energy, the wave length of the emitted radiation was found to be a decreasing function of the nanotube radius while the maximum number of bound states of the channeled positrons is an increasing function of the nanotube radius for the different types of SWCNTs namely, zigzag, armchair and chiral.

Values of the parameters a1, b1 and $\mathrm{c} 1$ in Eq. (7) needed to fit the nanotube channeling potential calculated by using different potential models for the given $(n, m)$ nanotubes, nmax is the maximum number of bound states for channeled positron with incident energy $100 \mathrm{MeV}$ incident in a direction parallel to the nanotube axis are presented in Table (1).

The maximum number of bound states nmax as calculated from Eq. (4) for different types of single wall carbon nanotubes (n,m) (armchair, chiral, zigzag)with energies (10-500) MeV in direction parallel to the nanotube axis are presented in Table (1), it is been found that, nmax is increasing with the radius in the same chiral indices $(n, m)$ of single wall carbon nanotubes.

The calculated results the energy of the emitted channeling radiation channeled positrons with $(\mathrm{n}, 0)$ (zigzag) of single wall carbon nanotubes by using Moliere potential at energies (10 MeV) were listed inTable (2).Also the calculated results of the wave length of the emitted channeling radiation channeled positrons with different chiral indices $(n, n)$ (armchair) of single wall carbon nanotubes by using Moliere potential at energies $(50 \mathrm{MeV})$ are given in from Table (3).

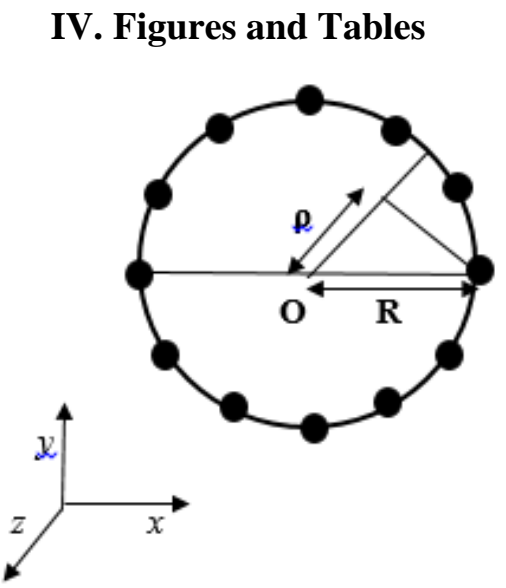

Fig. 1. The cross section view of $(6,0)$ nanotube is a circle of radius $R$, and $\rho$ is the distance measured from the center $\mathrm{O}$ in the $x y$-plane.

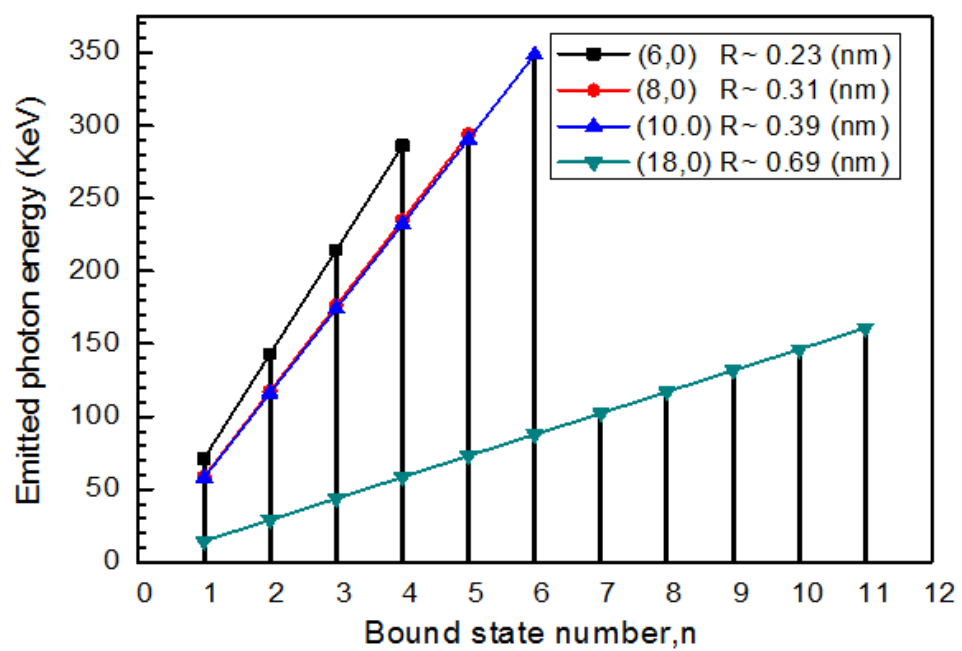

Fig. 2. The emitted photon energy due to the allowed transitions $n, n-1$ for positrons with $E=50 \mathrm{MeV}$ channeled in zigzag single-walled carbon nanotube selected with different diameters. 


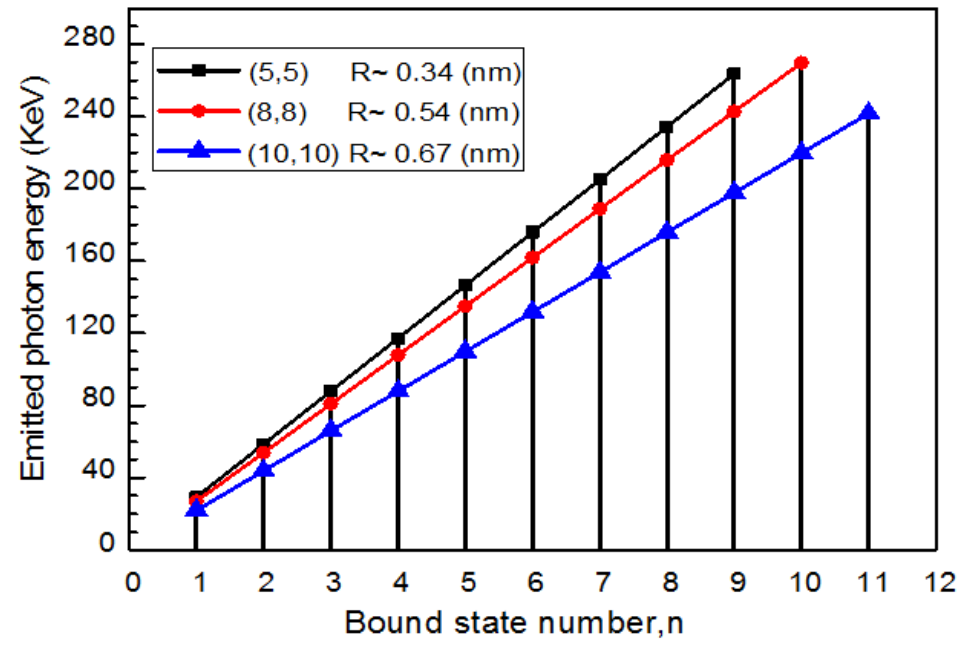

Fig. 3. The emitted photon energy due to the allowed transitions $n, n-1$ for positrons with $E=50 \mathrm{MeV}$ channeled in armchair single-walled carbon nanotube selected with different diameters.

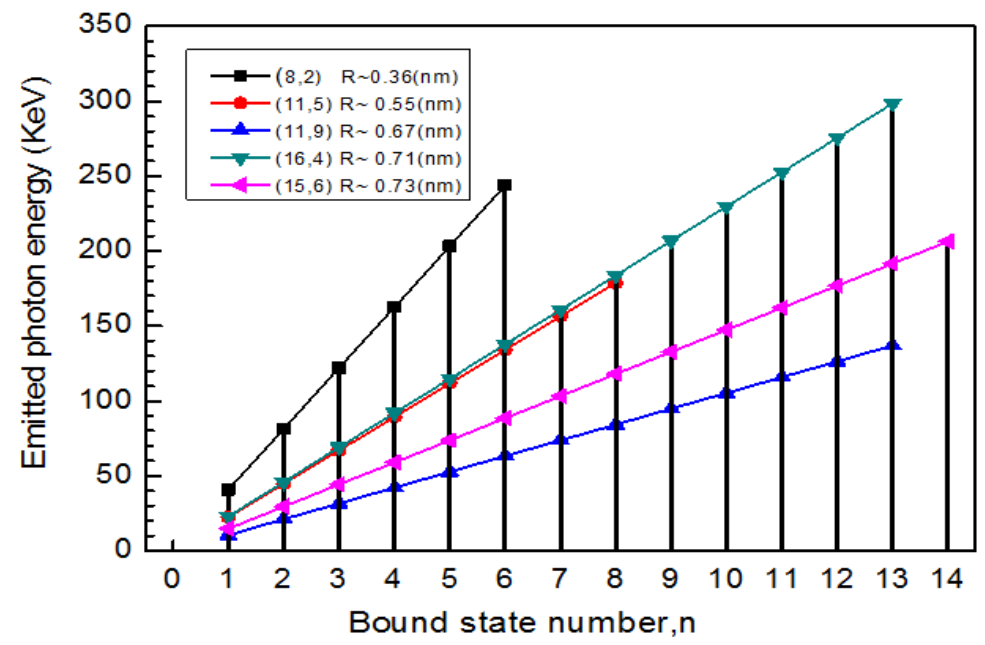

Fig. 4. The emitted photon energy due to the allowed transitions $n, n-1$ for positrons with $E=50 \mathrm{MeV}$ channeled in chiral single-walled carbon nanotube selected with different diameters.

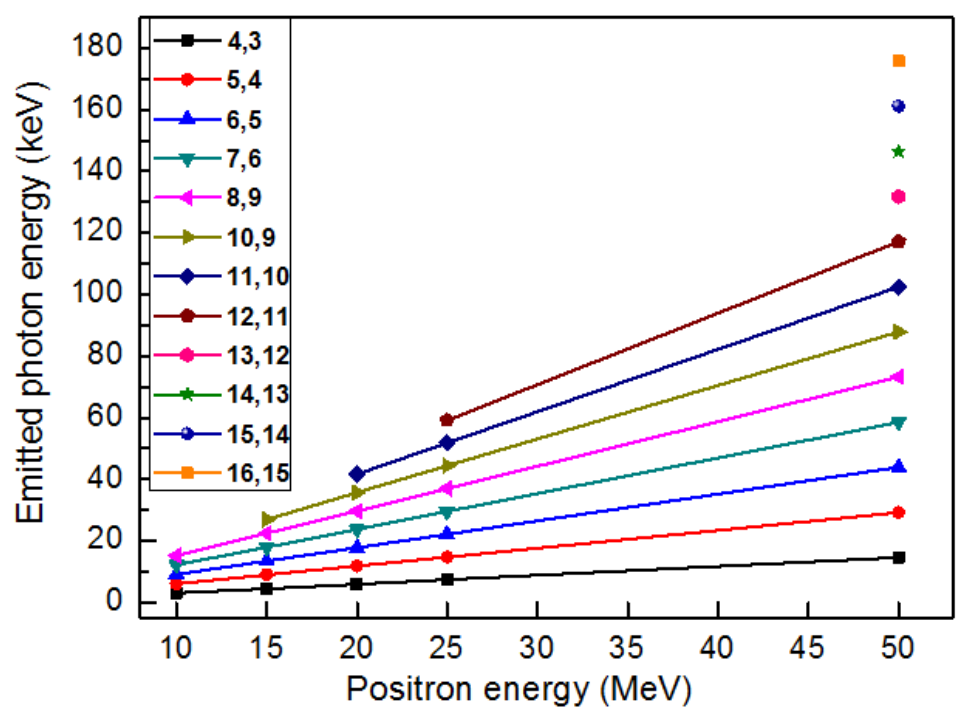

Fig. 5. The emitted photon energy in the forward direction due to $n, n-1$ transitions between higher quantum states of transverse motion in single- wall carbon nanotube $(18,0)$ as a function of positron energy up to 50 $\mathrm{MeV}$. 


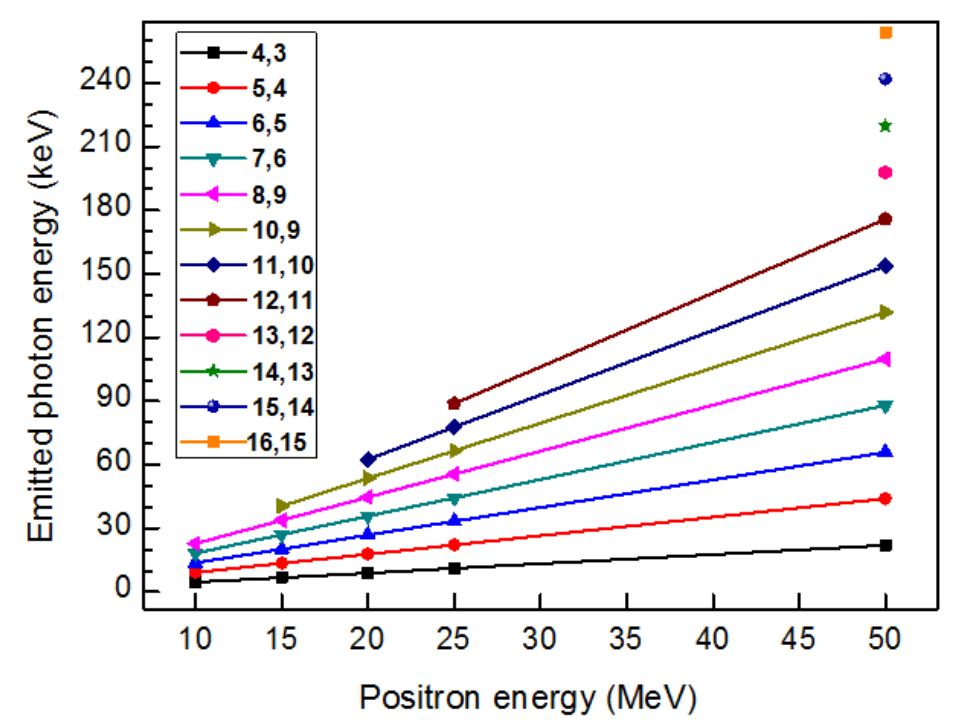

Fig. 6. The emitted photon energy in the forward direction due to $n, n-1$ transitions between higher quantum states of transverse motion in for single- wall carbon nanotube (10,10) as a function of positron energy up to 50 $\mathrm{MeV}$.

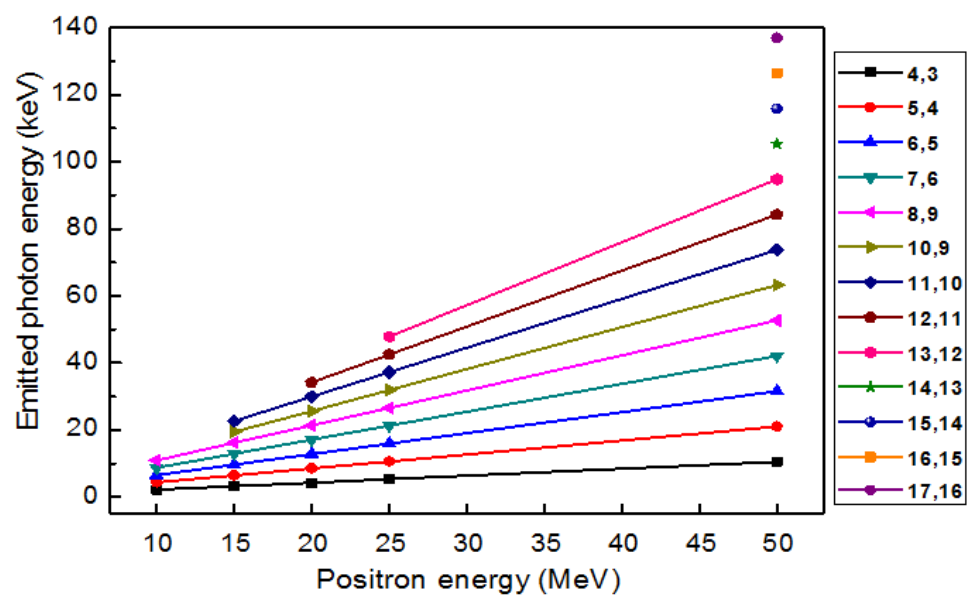

Fig. 7. The emitted photon energy in the forward direction due to $n, n-1$ transitions between higher quantum states of transverse motion in for single- wall carbon nanotube $(11,9)$ as a function of positron energy up to 50 $\mathrm{MeV}$.

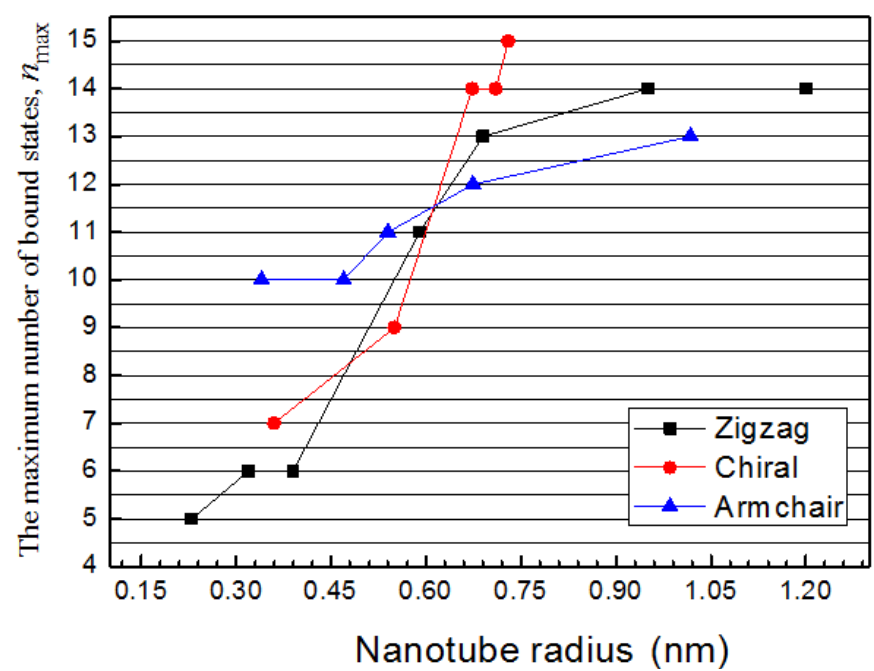

Fig. 8. The relation between the radius and the maximum number of bound state, $n_{\max }$ in (zigzag, chiral, armchair) single wall carbon nanotubes at $50 \mathrm{MeV}$. 


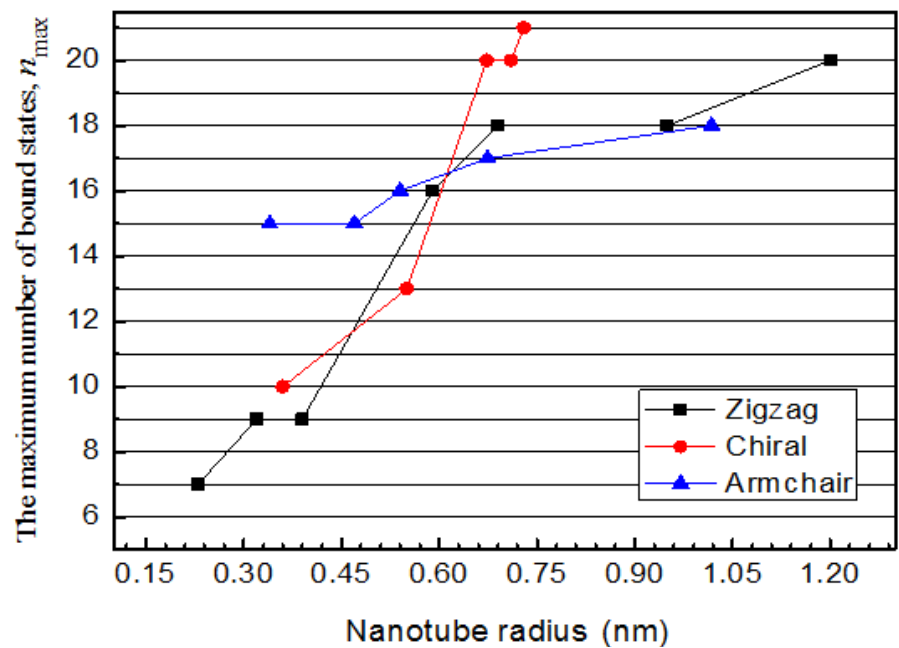

Fig. 9. The relation between the radius and the maximum number of bound state, $\mathrm{n}_{\max }$ in (zigzag, chiral, armchair) single wall carbon nanotubes at $100 \mathrm{MeV}$.

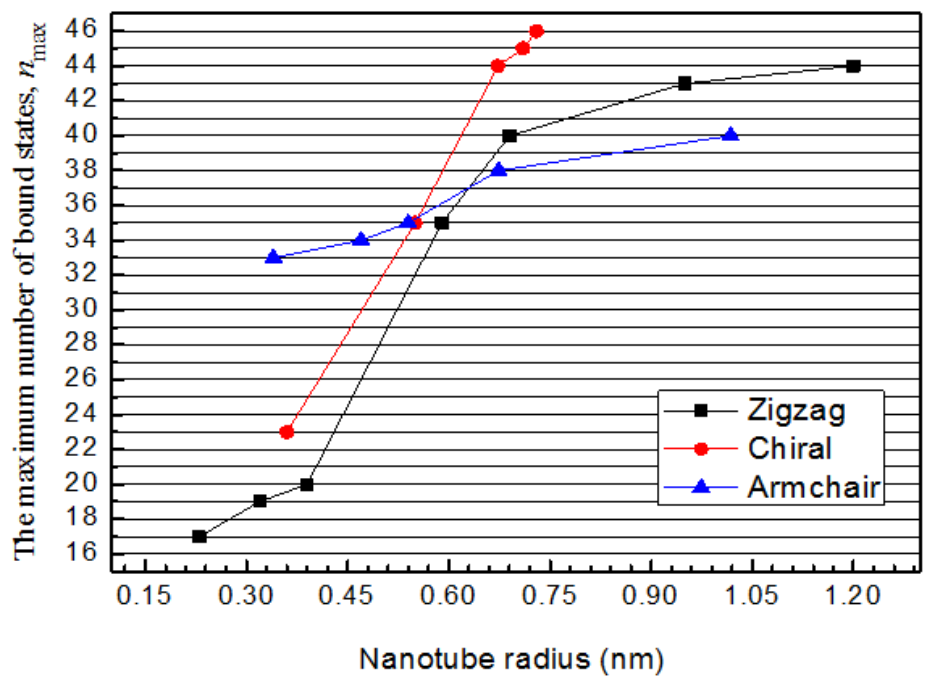

Fig. 10. The relation between the radius and the maximum number of bound state, $\mathrm{n}_{\max }$ in (zigzag, chiral, armchair) single wall carbon nanotubes at $500 \mathrm{MeV}$.

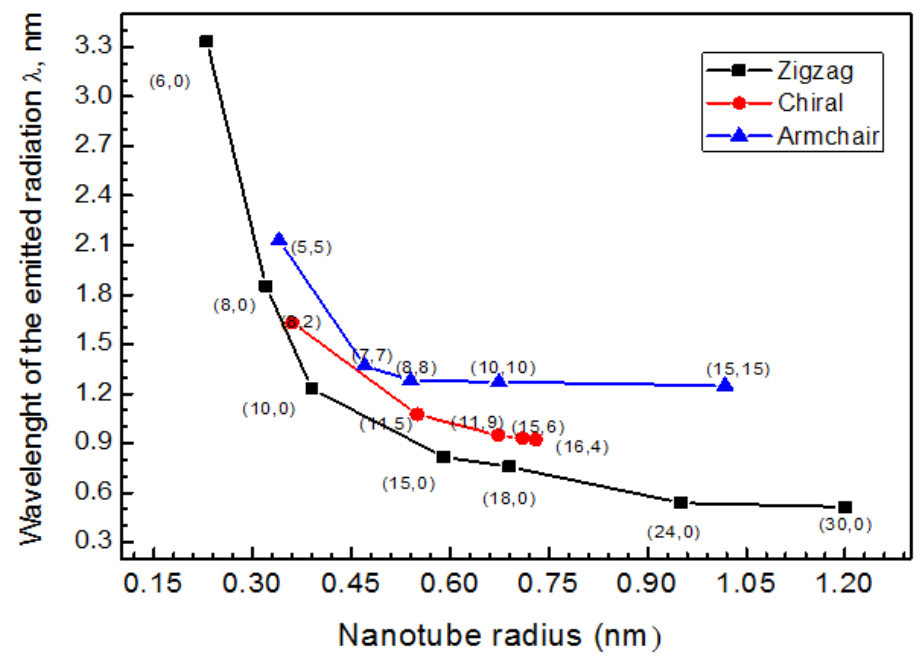

Fig. 11. The relation between the radius and wavelength in (zigzag, chiral, armchair) single wall carbon nanotubes at $50 \mathrm{MeV}$. 


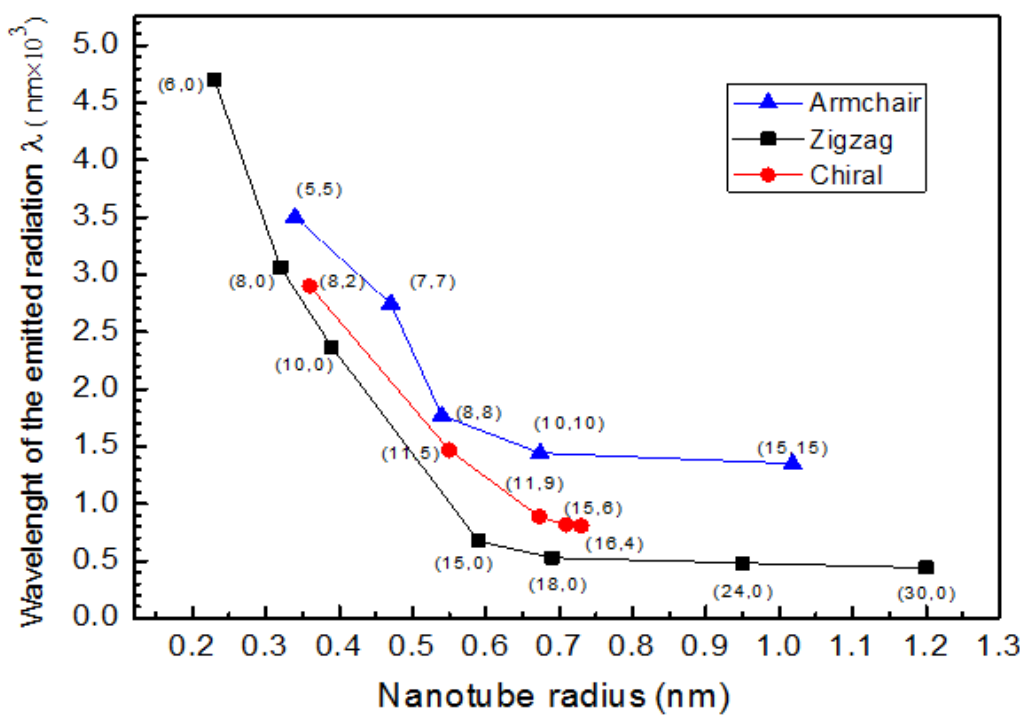

Fig. 12. The relation between the radius and wavelength in (zigzag, chiral, armchair) single wall carbon nanotubes at $100 \mathrm{MeV}$.

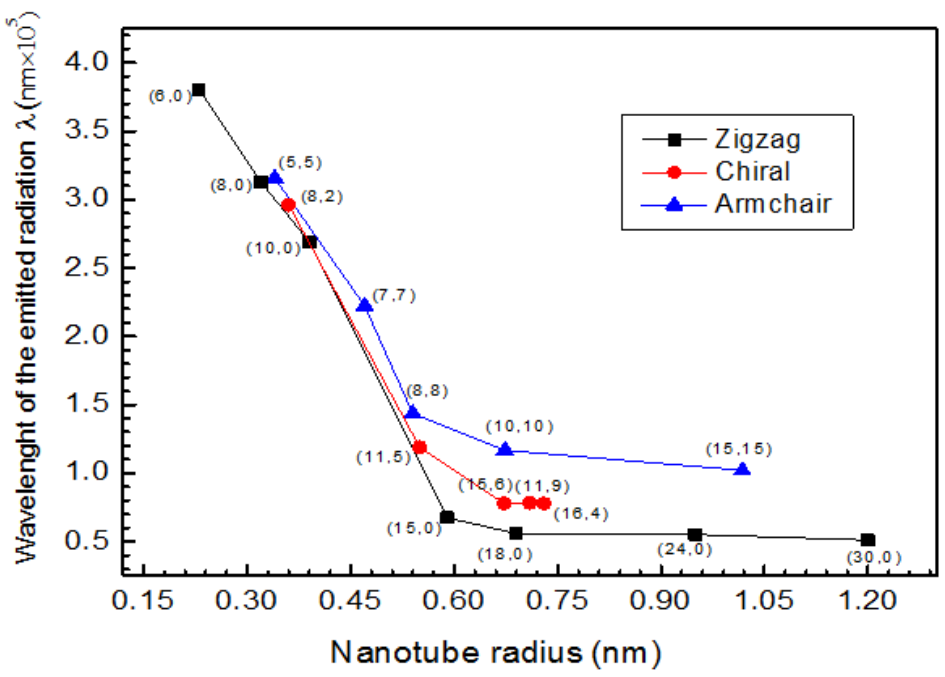

Fig. 13. The relation between the radius and wavelength in (zigzag, chiral, armchair) single wall carbon nanotubes at $500 \mathrm{MeV}$.

Table 1: Values of the parameters $a_{1}, b_{1}$ and $c_{1}$ in Eq. (7) needed to fit the nanotube channeling potential calculated by using different potential models for the given $(n, m)$ nanotubes, $n_{\max }$ is the maximum number of bound states for channeled positron with incident energy $100 \mathrm{MeV}$ incident in a direction parallel to the

\begin{tabular}{|l|l|l|l|l|l|}
\hline$(n, m)$ & Radius $(\mathrm{nm})$ & $a_{1}(\mathrm{eV})$ & $b_{1}(\mathrm{eV})$ & $c_{1}\left(\mathrm{~nm}^{-1}\right)$ & $n_{\max }$ \\
\hline$(5,5)$ & 0.337 & 6.78368 & 0.19424 & 19.46302 & 10 \\
$(8,8)$ & 0.483 & 1.90496 & 0.00246 & 23.2599 & 11 \\
$(15,15)$ & 1.031 & 0.66262 & $5.48 \times 10^{-10}$ & 26.4905 & 13 \\
\hline$(8,2)$ & 0.356237 & 4.10477 & 0.0281 & 22.92958 & 7 \\
$(11,9)$ & 0.67437 & 0.29904 & 0.0269 & 11.66859 & 14 \\
$(15,6)$ & 0.728205 & 0.15415 & 0.00492 & 13.80406 & 15 \\
\hline$(6,0)$ & 0.2332 & 12.0991 & 0.0932 & 30.40485 & 5 \\
$(15,0)$ & 0.5954 & 0.85968 & 0.0612 & 10.7511 & 11 \\
$(30,0)$ & 1.1909 & 0.07667 & $4.32 \times 10^{-5}$ & 12.08853 & 14 \\
\hline
\end{tabular}


Table 2: The maximum number of bound states ,nmax for channeling positrons in single wall carbon nanotubes(Chiral) different values for positron energy by using WKB approximation

\begin{tabular}{|r|r|r|r|r|r|r|r|r|}
\hline Positron energy(MeV) & 10 & 15 & 20 & 25 & 50 & 100 & 200 & 500 \\
\hline $\mathrm{n}_{\max }$ for(8,2) & 2 & 3 & 4 & 4 & 6 & 9 & 13 & 22 \\
$(\mathrm{R}=0.356237 \mathrm{~nm})$ & & & & & & & & \\
\hline $\mathrm{n}_{\max }$ for $(11,5)$ & 3 & 4 & 5 & 6 & 8 & 12 & 17 & 34 \\
$(\mathrm{R}=0.551059 \mathrm{~nm})$ & & & & & & & & \\
\hline $\mathrm{n}_{\max }$ for(11,9) & 5 & 7 & 8 & 9 & 13 & 19 & 27 & 43 \\
$(\mathrm{R}=0.674347 \mathrm{~nm})$ & & & & & & & & \\
\hline $\mathrm{n}_{\max }$ for $(16,4)$ & 6 & 7 & 8 & 9 & 13 & 19 & 27 & 44 \\
$(\mathrm{R}=0.712475 \mathrm{~nm})$ & & & & & & & & \\
\hline $\mathrm{n}_{\max }$ for $(15,6)$ & 6 & 7 & 8 & 9 & 14 & 20 & 28 & 45 \\
$(\mathrm{R}=0.728205 \mathrm{~nm})$ & & & & & & & & \\
\hline
\end{tabular}

Table3: The energy of the emitted channeling radiation channeled positrons at $10 \mathrm{MeV}$ in Zigzag for the transitions $n,(n-1)$.

\begin{tabular}{|c|c|c|c|c|c|c|c|}
\hline \multirow{2}{*}{$\begin{array}{r}\text { Allowed } \\
\text { transitions } \\
n, n-1\end{array}$} & \multicolumn{6}{|c|}{ Energy of the emitted radiation (KeV) } & \multirow[b]{2}{*}{$\begin{array}{l}(30,0) \\
n_{\max }=6\end{array}$} \\
\hline & $\begin{array}{r}(6,0) \\
n_{\max }=2\end{array}$ & $\begin{array}{r}(8,0) \\
n_{\max }=2\end{array}$ & $\begin{array}{l}(10,0) \\
n_{\max }=3\end{array}$ & $\begin{array}{l}(15,0) \\
n_{\max }=5\end{array}$ & $\begin{array}{c}(18,0) \\
n_{\max }=6\end{array}$ & $\begin{array}{l}(24,0) \\
n_{\max }=6\end{array}$ & \\
\hline $1 \rightarrow 0$ & 14.880 & 12.235 & 12.089 & 1.860 & 3.047 & 2.167 & 2.352 \\
\hline $2 \rightarrow 1$ & 29.761 & 24.470 & 24.178 & 3.721 & 6.094 & 4.335 & 4.704 \\
\hline $3 \rightarrow 2$ & No transition & No transition & 36.267 & 5.581 & 9.141 & 6.502 & 7.056 \\
\hline $4 \rightarrow 3$ & No transition & No transition & No transition & 7.442 & 12.189 & 8.670 & 9.408 \\
\hline
\end{tabular}

Table4: The wavelength $(\lambda)$ of the emitted channeling radiation channeled positrons at $50 \mathrm{MeV}$ in Armchair

\begin{tabular}{|c|c|c|c|c|c|}
\hline \multirow{2}{*}{$\begin{array}{r}\text { Allowed } \\
\text { transitions } \\
n, n-1\end{array}$} & \multicolumn{5}{|c|}{ The wavelength $(\lambda)$ of the emitted radiation $(\mathrm{nm})$} \\
\hline & $\begin{array}{r}(5,5) \\
n_{\max }=10\end{array}$ & $\begin{array}{r}(7,7) \\
n_{\max }=10\end{array}$ & $\begin{array}{r}(8,8) \\
n_{\max }=11\end{array}$ & $\begin{array}{l}(10,10) \\
n_{\max }=12\end{array}$ & $\begin{array}{l}(15,15) \\
n_{\max }=13\end{array}$ \\
\hline $1 \rightarrow 0$ & $1.516 \times 10^{-2}$ & $2.165 \times 10^{-2}$ & $1.396 \times 10^{-2}$ & $1.1369 \times 10^{-2}$ & $3.116 \times 10^{-2}$ \\
\hline $2 \rightarrow 1$ & $3.032 \times 10^{-2}$ & $4.33 \times 10^{-2}$ & $2.792 \times 10^{-2}$ & $2.2739 \times 10^{-2}$ & $5.616 \times 10^{-2}$ \\
\hline $3 \rightarrow 2$ & $4.548 \times 10^{-2}$ & $6.495 \times 10^{-2}$ & $4.188 \times 10^{-2}$ & $3.411 \times 10^{-2}$ & $8.425 \times 10^{-2}$ \\
\hline $4 \rightarrow 3$ & $6.064 \times 10^{-2}$ & $8.66 \times 10^{-2}$ & $5.584 \times 10^{-2}$ & $4.548 \times 10^{-2}$ & $1.123 \times 10^{-1}$ \\
\hline $5 \rightarrow 4$ & $7.579 \times 10^{-2}$ & $1.083 \times 10^{-1}$ & $6.979 \times 10^{-2}$ & $5.685 \times 10^{-2}$ & $1.404 \times 10^{-1}$ \\
\hline $6 \rightarrow 5$ & $9.095 \times 10^{-2}$ & $1.299 \times 10^{-1}$ & $8.376 \times 10^{-2}$ & $6.822 \times 10^{-2}$ & $1.685 \times 10^{-1}$ \\
\hline $7 \rightarrow 6$ & $1.0612 \times 10^{-}$ & $1.516 \times 10^{-1}$ & $9.772 \times 10^{-2}$ & $7.959 \times 10^{-2}$ & $1.966 \times 10^{-1}$ \\
\hline $8 \rightarrow 7$ & $1.213 \times 10^{-1}$ & $1.732 \times 10^{-1}$ & $1.117 \times 10^{-1}$ & $9.096 \times 10^{-2}$ & $2.246 \times 10^{-1}$ \\
\hline $9 \rightarrow 8$ & $1.3644 \times 10^{-}$ & $1.949 \times 10^{-1}$ & $1.256 \times 10^{-1}$ & $1.023 \times 10^{-1}$ & $2.527 \times 10^{-1}$ \\
\hline $10 \rightarrow 9$ & $1.5159 \times 10^{-}$ & $2.165 \times 10^{-1}$ & $1.396 \times 10^{-1}$ & $1.137 \times 10^{-1}$ & $2.808 \times 10^{-1}$ \\
\hline $11 \rightarrow 10$ & No transition & No transition & $1.5356 \times 10^{-1}$ & $1.251 \times 10^{-1}$ & $3.089 \times 10^{-1}$ \\
\hline $12 \rightarrow 11$ & No transition & No transition & No transition & $1.364 \times 10^{-1}$ & $3.369 \times 10^{-1}$ \\
\hline $13 \rightarrow 12$ & No transition & No transition & No transition & No transition & $3.651 \times 10^{-1}$ \\
\hline
\end{tabular}

\section{Conclusion}

This study covers SWCNTs with different chiral indices $(n, m)$. The energy eigenvalues, in a plane normal to the nanotube axis (xy-plane), of relativistic positron channeled through SWCNTs has been used to calculate the emitted photon energy in the forward direction, i.e., channeling radiation. The calculations covered both the wave length of the emitted radiation and the maximum number of bound states of the channeled positrons as a function of the nanotube radius at different incident energies. The results of the calculations showed that, in this energy range, the emitted radiation energy lies in the X-ray band and as the energy of the incident positron increase, the emitted radiation energy increase towards the gamma-ray energy band and accordingly, the wave length decrease towards the gamma-ray band for the same transition $\Delta E=E_{n+1}-E_{n}$.

\section{References}

[1] M. A. Kumakhov, Phys. Lett. 57A, 17,1976, Dokl.Akad.SSSR,230,1077,1976.

[2] M. A. Kumakhov, Zh.Eksp. Teor. Fiz. 72, 1489,1977, phys. stat. solidi (b),,1977,84-41, and phys. stat. solidi (b), 84,581 ,1977.

[3] V.V.Klimov and V.S.Letokhov. "Monochromatic Radiation emitted by a relativistic electron moving in a carbon nanotube", Physics Letters A, 226 1997, 244-252.

[4] G.V. Dedkov, "Fullerene nanotubes can be used when transporting gamma-quanta, neutrons, ion beams and radiation from relativistic particles", Nucl.Instrum.Methods B 1435841998

[5] M. K. Abu-Assy, M.Y.El-Ashry, A. R. Abdullah and M. H. Ali. " Energy spectrum of relativistic positrons channeled through single-walled carbon nanotubes". Accepted for publication in Egypt. J. Solids.2016.

[6] J. Lindhard, "Influence of crystal lattice on motion of energetic charged particle", Mat. Fys. Medd. Dan. Vid. Selsk. 34 (14) 1965. 
[7] G. Moliere, Z. Naturforsch. A2, 133, 1947.

[8] L. I. Schiff, "Quantum Mechanics", New York, Mc Graw-Hill, 1968.

[9] R. H. Pantell and R. L. Swent, Appl. Phys. Lett. 35(12), 197.

[10] S. Satpathy and A. P. Pathak, "Planar Channeling of Electrons and Positrons in Crystals", phys. stat. sol. (b) 153, 455 1989. 